\title{
Article \\ Emerging Quantum Fields Embedded in the Emergence of Spacetime
}

\section{Hans Diel}

Diel Software Beratung und Entwicklung, Seestr.102, 71067 Sindelfingen, Germany, diel@netic.de

\begin{abstract}
Based on a local causal model of the dynamics of curved discrete spacetime, a causal model of quantum field theory in curved discrete spacetime is described. At the elementary level, space(-time) is assumed to consists of interconnected space points. Each space point is connected to a small discrete set of neighbor space points. Density distribution of the space points and the lengths of the space point connections depend on the distance from the gravitational sources. This leads to curved spacetime in accordance with general relativity. Dynamics of spacetime (i.e., the emergence of space and the propagation of space changes) dynamically assigns "in-connections" and "out-connections" to the affected space points. Emergence and propagation of quantum fields (including $\mathrm{p}$ articles) a re $\mathrm{m}$ apped to the e mergence and p ropagation of $\mathrm{s}$ pace changes by utilizing identical paths of in/out-connections. Compatibility with standard quantum field theory (QFT) requests the adjustment of the QFT techniques (e.g., Feynman diagrams, Feynman rules, creation/annihilation operators), which typically apply to three in/out connections, to $n>3$ in/out connections. In addition, QFT computation in position space has to be adapted to a curved discrete space-time.
\end{abstract}

Keywords: quantum field theory, local causal models, general relativity theory, spacetime models, discrete spacetime, computer simulations

\section{Introduction}

The authors attempt to construct a local causal model of quantum theory (QT), including quantum field theory (QFT), soon resulted in the recognition that a causal model of the dynamics of QT/QFT should better be based on a causal model of the dynamics of spacetime. Thus, a causal model of the dynamics of spacetime has been developed with the major goals (1) as much as possible compatibility with general relativity theory (GRT), and (2) the model should match the main features of the evolving model of QT/QFT. The main features of the authors model of QT/QFT are

- the model has to be a causal model,

- if possible, the model should be a local causal model,

- discreteness of the basic parameters (time, space, propagation paths).

Not surprisingly, it turned out that a clear definition of these features/requirements, especially of a local causal model, is useful (not only for understanding the requirements, but also for the derivation of the implications). A semi-formal definition of a (local) causal model has been published in several articles from the author (see [1], [2] and [3]) and is also given in Section 2.

The construction of a causal model of spacetime dynamics started with the search for some existing theory or model which might be at least a starting point for the model to be developed. Causal dynamical triangulation (CDT, see [4], [5], [6]) and more abstractly the concepts of loop quantum gravity (see [7] and [8]) were identified to match the authors requirements and thinking. The further model construction showed that, in order to come up with a local causal model according to the definitions given in Section 2, adaptations and refinements of the original CDT-based model appear appropriate. The adaptations and refinements concern basic GRT concepts such as (i) the elementary structure of space(-time), (ii) the representation of space(-time) curvature, and (iii) the relation between 
space and time. With GRT and special relativity theory (SRT), space and time are said to be integrated into spacetime. For the GRT-compatible model of spacetime dynamics, the integration of space and time remains, but with a different interpretation. The elementary structure of space(-time), including the space-time relationship is described in Section 3. The causal model of the spacetime dynamics is described in Section 4.

The major goal for the development of a causal model of spacetime dynamics (Sections 3 and 4) was to develop a model of the spacetime elementary structure that constitutes a suitable base for both the causal model of spacetime dynamics and the causal model of QT/QFT. The proposed model satisfies this goal. The emergence and propagation of quantum fields (including particles) can be mapped to the emergence and propagation of space changes by utilizing identical paths of in/out-connections between space points. In Section 5, this main subject of the article is described.

\section{Causal Models}

The specification of a causal model of a theory of physics consists of (1) the specification of the system state, (2) the specification of the laws of physics that define the possible state transitions when applied to the system state, and (3) the assumption of a "physics engine."

\subsubsection{The physics engine}

The physics engine represents the overall causal semantics of causal models. It acts upon the state of the physical system. The physics engine continuously determines new states in uniform time steps. For the formal definition of a causal model of a physical theory, a continuous repeated invocation of the physics engine is assumed to realize the progression of the state of the system.

physics engine $(S, \Delta t):=\{$

DO UNTIL(nonContinueState $(S))\{$

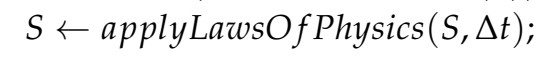

$$
\}
$$

\section{\}}

\subsubsection{The system state}

The system state defines the components, objects and parameters of the theory of physics that can be referenced and manipulated by the causal model. In contrast to the physics engine, the structure and content of the system state are specific for the causal model that is being specified. Therefore, the following is only an example of a possible system state specification.

systemstate $:=\{$ spacepoint... $\}$

spacepoint $:=\left\{x_{1}, x_{2}, x_{3}, \psi\right\}$

$\psi:=\left\{\right.$ stateParameter $_{1}, \ldots$, stateParameter $\left._{n}\right\}$

\subsubsection{The laws of physics}

The refinement of the statement

$S \leftarrow$ applyLawsOfPhysics $(S, \Delta t)$; defines how an "in" state s evolves into an "out" state s.

$L_{1}:=I F c_{1}(s)$ THEN $s \leftarrow f_{1}(s) ;$

$L_{2}:=I F c_{2}(s)$ THEN $s \leftarrow f_{2}(s) ;$

...

$L_{n}:=\operatorname{IF} c_{n}(s) T H E N s \leftarrow f_{n}(s) ;$

The "in" conditions $c_{i}(s)$ specify the applicability of the state transition function $f_{i}(s)$ in basic formal (e.g., mathematical ) terms or refer to complex conditions that then have to be refined within the formal definition. 
The state transition function $f_{i}(s)$ specifies the update of state $s$ in basic formal (e.g., mathematical) terms or refers to complex functions that then have to be refined within the formal definition.

The set of laws $L_{1}, \ldots, L_{n}$ has to be complete, consistent and reality conformal (see [9] for more details).

In addition to the above-described basic forms of specification of the laws of physics by $L_{n}:=$ $I F c_{n}(s)$ THEN $s \leftarrow f_{n}(s)$, other forms are also imaginable and sometimes used in this article. ${ }^{1}$

\subsection{Requirements for causal models of spacetime}

For causal models of spacetime, obviously, some notion of space and time must be supported. Ideally, the treatment of space and time would be, as much as possible, compatible with special relativity theory (SRT) and general relativity theory. However, the formally defined causal model of Section 2 presupposes a certain structure of spacetime in which space and time are rigorously separated. This disturbs the integrated view of space and time that is taught by GRT/SRT. In the proposed model of spacetime dynamics, the integration of space and time is largely restored by the specification of the relationships described in Section 3.1.

\subsubsection{The representation of time in the causal model}

In the causal model defined above, time is not like space and other parameters a system state component, but it has a special role outside the system state. The overall purpose of the causal model is seen in showing the progression of the system state in relation to the progression of time. This relationship can best be described by assuming a uniform progression of the time. This leads to the model (described above) where the time and the progression of time is built into the model in the form of the physics engine. The physics engine progresses the system state in uniform time steps called state update time intervals (SUTI).

In GRT and SRT, there are situations where the clock rate of a causal subsystem is predicted to differ depending on the relative speed of movement or the position within a gravitational field. GRT and SRT refer to this by the name "proper time". If, for a specific causal model of an area of physics the differing proper times of causal subsystems are relevant and/or the internal processes within the subsystems are included in the model, separate physics engines may be assigned to the subsystems with different proper times. ${ }^{2}$

If, however, the causal model describes an area of physics where the relationship between proper times and other parameters is to be shown, it should be possible to show this with a single physics engine and a uniform SUTI for the overall system. For the proposed causal model of spacetime dynamics, the space-time relationship described in Section 3.1 enables a single physics engine and a uniform SUTI.

\subsubsection{Spatial causal model}

A causal model of a theory of physics is called a spatial causal model if (1) the system state contains a component that represents a space, and (2) all other components of the system state can be mapped to the space. There exist many textbooks on physics (mostly in the context of relativity theory) and mathematics that define the essential features of a "space". For the purpose of the present article, a more detailed discussion is not required. For the purpose of this article and the subject locality, it is sufficient to request that the space (assumed with a spatial model) supports the notions of position, coordinates, distance, and neighborhood.

1 This article does not contain a proper definition of the used causal model specification language. The language used is assumed to be largely self-explanatory.

2 An example can be found in the causal model described in [3], where separate physics engines are assigned to the "quantum objects". 
A special type of spatial causal model that has been increasingly addressed in recent years is the cellular automaton (see [10], [11], [12] and [13]). The causal model described in this article also represents a spatial causal model.

\subsubsection{Local causal model}

The definition of a local causal model presupposes a spatially causal model (see above). A (spatially) causal model is understood to be a local model if changes in the state of the system depend on the local state only and affect the local state only. The local state changes can propagate to neighboring locations. The propagation of the state changes to distant locations; however, they must always be accomplished through a series of state changes to neighboring locations. ${ }^{3}$

Based on a formal model definition of a causal model, a formal definition of locality can be given. A physical theory and a related spatially causal model with position coordinates $\mathrm{x}$ and position neighborhood $\mathrm{dx}$ (or $\Delta x$ in the case of discrete space-points) are given. A causal model is called a local causal model if each of the laws $L_{i}$ applies to no more than a single position $\mathrm{x}$ and/or to the neighborhood of this position $x \pm d x$.

In the simplest case, this arrangement means that $L_{i}$ has the form

$L_{i}: \operatorname{IF} c_{i}(s(x)) \operatorname{THENs}^{\prime}(x)=f_{i}(s(x))$;

The position reference can be explicit (for example, with the above simple case example) or implicit by reference to a state component that has a well-defined position in space. References to the complete space of a spatially extended object or to a property of a spatially extended object are considered to violate "space-point-locality". Causal models with a system state that includes composite objects with global properties (e.g., mass, charge, velocity) may still be considered local causal models, more specifically "object-local causal model", even if such global properties are referenced in the model.

\subsubsection{Background-independence}

Background independence is an important requirement that is typically established for spacetime models such as spin networks, spin foam, and causal dynamical triangulation. This requirement seems to be mandatory for a local causal spacetime model that supports the emergence of spacetime from a minimal or zero source. Background independence means that all spacetime dynamics, in particular the emergence of space, must be expressible without reference to any predefined coordinate system or other global spacetime properties. For a causal model, this means that the structure of spacetime must not contain components and properties that are non-local.

\subsubsection{Composite objects}

Models of areas of physics typically contain spatially extended composite objects such as particles, atoms, stars, and so forth, and typically object-global properties (e.g., mass, charge, velocity) are referenced in such models. According to the definition of a local causal model (above), such models may only be called "object-local causal models" (as opposed to "space-point-local causal models"). Such models may be useful; however, care must be taken that the assignment of object-global properties to composite objects is admissible with the level of accuracy aimed for. Object-global properties are typically the result of aggregations from lower-level relationships. The aggregations toward a single global attribute value may be admissible with classical physics, but questionable with refinements of modern theories of physics. A famous example of the inclusion of global object properties refers to the attributes of mass and charge with quantum field theory when particles are no longer considered to be point-like particles.

3 Special relativity requests that the series of state changes does not occur with a speed that is faster than the speed of light. This requirement is not considered essential for a causal model. 


\section{The elementary structure of spacetime}

\subsection{The space-time relationship}

With GRT and SRT, space and time are said to be integrated into spacetime. For a GRT-compatible model of spacetime dynamics, the integration of space and time remains visible, but with a different interpretation. With GRT, the integration of space and time is mathematically expressed in the usage of tensors (e.g., curvature tensor) and 4-vectors with a time component and space components. Physically, the integration is reflected, among other ways, in the metric and the symmetries that hold for the combined (space+time) entities and the corresponding laws of physics.

In the proposed causal model of spacetime dynamics, the tensors and 4-vectors of GRT/SRT occur only as the starting point for the introduction of GRT-compatible equivalent model parameters. The integration of space and time appears to be disturbed by the fundamentally different roles space and time represent in a causal model. Time and the progression of time are an inherent feature of the physics engine of the causal model. The physics engine implements the uniform and simultaneous progression of time. Space is the explicit global object that is part of the system state. Other objects of the system state are positioned in space. Although space and time conceptually have quite different roles within the causal model, it is their mutual relationship that establishes their (re-)integration.

In GRT, the curvature specification, i.e., the curvature tensor, contains, in addition to the three space-related components, a time-related component. As an example of the impact of the time factor, the gravitational redshift is explained as the consequence of the time factor in the spacetime curvature (see, for example, [14], page 231).

$$
\Delta s^{2}=-\left(1-\frac{2 G M}{c^{2} r}\right)(c \Delta t)^{2}+(\Delta x)^{2}+(\Delta y)^{2}+(\Delta z)^{2}
$$

This means a clock at position $(\mathrm{x}, \mathrm{y}, \mathrm{z})$ would run by a factor

$$
F_{1}=\sqrt{1-\frac{2 G M}{c^{2} r}}
$$

slower than a clock that is not affected by a gravitational field. A standard clock at some point A of low potential (for example, on the surface of the earth) would go slower than the same clock at point B of higher potential (for example, at a GPS satellite). In [14]: "... The gravitational redshift implies that time itself runs slightly faster at the higher altitude than it does on the Earth." For the GPS system, the difference is 45 microseconds per day: This is the rate at which the clocks at the satellites go faster (see [15]). In GRT, this effect is called "gravitational time dilation". For reasons that are described in the following, the author prefers the wording (gravitational) "clock rate dilation".

For a mapping of the time factor of the GRT curvature specification to the proposed spacetime model, two problems arise:

1. In the causal model, the clock rate (i.e., the proper time) is a property of the whole causal subsystem. The assignment of clock rates to the different positions occupied by a spatial distributed causal subsystem is not supported with the proposed causal model. ${ }^{4}$

2. In the causal model, the clock rate is maintained by the physics engine (i.e., the clock is part of the physics engine which delivers the uniform state update time interval). Changes in the clock rate resulting from the objects motion in space would mean that the clock of the physics engine has to run slower or faster depending on the object's position in space. This would require a rather ugly interface between the space and the physics engines of the causal subsystems.

4 The assignment of differing clock rates to the different positions occupied by a spatial distributed causal subsystem would make causal models for the dynamics of subsystems extremely difficult. 
Problem (2) may be viewed as a problem due to the specific definition of a causal model given in Section 2. However, there are (good) reasons for this definition of a causal model. Problem (1) refers to the causal model of causal subsystems in general. It would also be difficult to avoid this problem with alternative causal model concepts.

A possible solution that would make it possible to maintain a uniform progression of the state update time interval SUTI while enabling non-uniform clock rates may be found if one remembers that, in SRT and GRT, space and time are considered as an entity and that this implies that space intervals and time intervals can be jointly transformed by certain symmetry transformations. For the example gravitational redshift, this means that the redshift is interpreted as the dilation of the wave length instead of the increase of the frequency and that the length dilation affects not only the wave length but all lengths within the gravitational potential. For the proposed model of spacetime dynamics, it is assumed that

\section{Proposition 1. Lengths within the gravitational field are dilated by the factor $F_{1}$.}

${ }^{5}$ How can this help to prevent the need for the dynamic and position-dependent change of the state update time interval (SUTI)? A further proposition was introduced:

Proposition 2. Physical processes run faster/slower depending on the length scale at the position where the respective physical process executes.

Notice that the clock rate dilation concerns physical processes, not the spacetime structure. Space(-time) curvature is the result of length dilations. Clock rate dilation is another consequence of length dilations.

The major process that demonstrates the fixed relationship between the length dilation and the process change rate is the propagation of light. This (simple) process is used as a measure for the change rate of other processes by setting the speed of light to be a constant $c$. The next class of processes where the change rate depends on the length dilation in precisely the proportions as with the propagation of light are clocks in differing realizations.

In summary, in the model of spacetime dynamics, there is no direct reflection of time dilation as a spacetime attribute. Clock rate dilation (rather than time dilation) occurs as a property of processes running within space. The clock rate dilation factor can be derived from the length dilation factor $F_{1}$ of the space points where the respective process is currently executing.

In the model of spacetime dynamics, two levels of time are distinguished, which in GRT/SRT are seen as an entity:

1. At the basic level, the progression of time is associated with the physics engine of the causal model. The time of the physics engine proceeds in uniform state update time intervals. Simultaneousness is assumed for all state changes occurring at the same state update cycle.

2. Differing clock rates, proper times, and relativity of simultaneousness are not associated with the basic overall spacetime, level (1), but are associated with objects residing and moving in space more precisely, with processes running in these subsystems.

With space, two levels also may be distinguished, but these are two levels of consideration:

5 "Gravitational length dilation" appears to be a very controversial subject among physicists (see various discussion in internet forums). The author here takes a strong position while at the same time stating a clear relation between (1) the length dilation and (2) the clock rate dilation, namely by saying that (2) is a consequence of (1). 
- At the abstract level (i.e., mathematical level), the space consists of a set of interconnected space points (see Section 3). Whether or not the totality of interconnected space points represents an Euclidean space or a specific topology (e.g., Riemann manifold) is left open.

- At the physical level (i.e., the essential level), meaning is assigned to the components of the space point. Especially, the length of the connections is no longer a geometrical property, but specifies the $\Delta$ length only with respect to a specific physical process executing at the respective space point for the time interval SUTI. The process that is used as the measure for the specification of the length is the propagation of light.

Thus, the integration of space and time into spacetime is established in the model of spacetime dynamics by the physical meaning assigned to the components of the space points and their connections.

1. Time progresses uniformly in constant units. As a suitable basic unit of time progression, the state update time interval (SUTI) of the physics engine is taken. This means, the SUTI is assumed to be a system constant.

2. Length specification is expressed in relation to the spatial distance change caused by a specific physical process running for the duration of the standard unit of time (i.e., the SUTI). ${ }^{6}$

3. The physical process that is used as the measure for the standard unit of time as well as the measure of spatial distances is the propagation of light. ${ }^{7}$

The proposition (fact?) that there is such a simple relationship between the spatial length dilations and the rate of state changes of processes that execute at a given position in space is the root of the space-time integration in the proposed model of spacetime dynamics. A possible foundation of this supposed space-time relationship (reflecting the space-time integration) may be that

Conjecture 3.1. All physical processes can ultimately be broken down to length-related state changes, and changes in the length scaling therefore directly result in clock rate dilations of the affected process.

\subsection{The elementary structure of space}

The proposed elementary structure of spacetime constitutes the base for the overall model of spacetime dynamics that is compatible with GRT. A number of works toward the same or a similar goal have been published. The work that shows the most similarities with the model described in this article in terms of the overall orientation (background independence; discreteness of time, space, and paths; expressing causal relationships) is causal dynamical triangulation (CDT, see [4], [5], and [6]). The spacetime structure of the model described in this article is based on CDT. However, it was felt that adaptations were required to further refine the causal relationships of spacetime dynamics, in particular to construct a causal model of the emergence of space from a single source.

With CDT, the basic space elements are n-dimensional simplexes (e.g., triangles, tetrahedrons; see Fig. 1). In contrast to CDT, the proposed causal model of curved discrete spacetime considers only 3-dimensional space elements, i.e., tetrahedrons. The time dimension is treated separately within the causal model. In addition, the elementary units that represent the total space are not (as with CDT) the n-dimensional simplexes, but only the space points together with their connections to neighbor space points ${ }^{8}$. Whether the space points together with the connections establish specific 2-dimensional surface areas (e.g., triangles) and 3-dimensional solids (e.g., tetrahedrons) is initially left open.

6 This means, in the causal model, spatial distances are not primarily a geometrical property, but rather a physical property used to formulate interrelationships between objects in space.

7 This has the consequence, that in the model (as with most models of physics), the speed of light $\mathrm{c}$ is a constant.

8 The reason for this simplification was that it was not possible to build up a larger space object by the continuous addition of uniform regular tetrahedrons and (2) the uniformness of the tetrahedrons is obsolete with the proposed model (see Section $4)$. 


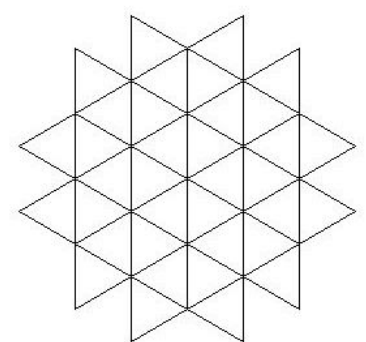

2-dimensional

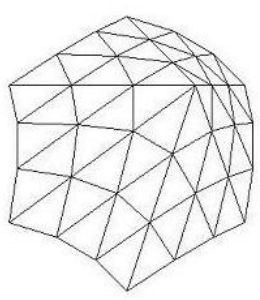

2-dimensional curved

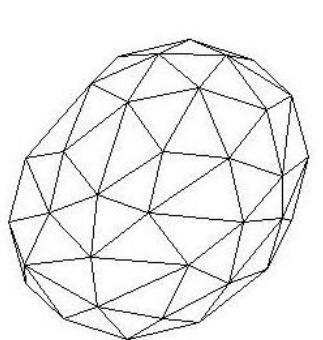

3-dimensional

Figure 1. Elements of spacetime of Causal Dynamical Triangulation.

\author{
Definition 1. Space $:=\{$ spacepoint $\ldots\}$; \\ spacepoint $:=\{\psi$, dilation factor, connections $\}$; \\ connections $:=\left\{\right.$ connection $_{1}, \ldots$, connection $\left._{n}\right\}$; \\ connection $:=\{$ neighborspacepoint, direction, $\Delta$ curvature $\}$;
}

$\psi$ is the physical content that is directly associated with the space. These are the fields residing in space. As with spin networks, spin foam networks, and causal dynamical triangulation, each space point is connected with a number of other space points via "connections" (i.e., edges in CDT). A connection carries the information about the connected neighbor space point, the connection direction, and the propagation gradient of the curvature changes (see Section 4).

All the information associated with the space point is local to the space point (i.e., no globally defined position or direction specification). This supports the background independence of the spacetime model.

To enable the determination of the spatial distance between two space points, some information about the distance between neighbor space points is required. This could be provided, for example, in form of position coordinates ${ }^{9}$ or by the specification of the lengths of connections between the neighbor space points. In support of a causal model of the movement of objects in curved space, for the proposed model of spacetime dynamics, it is defined that

\section{Proposition 3. The length of the connections between space points is a constant;}

$L_{\text {connection }}=c \cdot$ SUTI.

The overall distance between two space points within the curved space is then obtained by multiplying $L_{\text {connection }}$ by the number of space points $k_{p}$ on the geodesic path from space point- 1 to space point-2. Length dilation within a gravitational potential as assumed by Proposition 1 in Section 3.1 , is realized by the appropriate arrangement of the space points within space (see Section 4).

Proposition 3 is, first of all, a physical statement, although it has consequences for the space geometry. The physical statement is:

The (spatial) distance that light moves during a state update time interval (SUTI) is equal to the distance between two connected neighbor space points, which is equal to the distance by which space expands during a SUTI.

The geometry of the emerged space (e.g., whether an Euclidean space or a Schwarzschild metric emerges) depends on the space expansion algorithm. With the proposed model of spacetime dynamics

9 Provision of space point coordinates would violate background independence. 
the resulting geometry depends on the ratio by which the number of space points grow at a single expansion step (see Section 4.1).

\subsection{The representation of space(-time) curvature}

Space curvature is a major ingredient of GRT. In GRT, specifically in Einstein's equation $G^{\alpha \beta}=8 \pi T^{\alpha \beta}$, space curvature is expressed by the curvature tensor $G^{\alpha \beta}$. Thus, the simplest solution would be to say that a space-curvature component is assigned to the space point and that this curvature specification provides the same information as the curvature tensor of GRT. However, some adaptations appear reasonable. In Section 3.2 above, the space component of the system state is specified as consisting of a set of space points, and, at the next level of detail, a space point is specified as consisting of dilationfactor, connections, and the space content $\psi$.

spacepoint $:=\{\psi$, dilationfactor, connections $\}$;

The dilationfactor supports the generation of the space curvature with the propagation of space changes (including the emergence of space). Once the space has emerged, the space(-time) curvature is represented by (1) the distribution and density of the space points and (2) the (spatial) distances between neighboring space points. Proposition 3 (above) states that the length of the connections between space points, i.e., the distances between neighboring space points, is a constant. Thus, the main parameter that determines the space curvature is the density distribution of the space points. The density distribution of space points is realized by the appropriate arrangement of the space points within space.

As described in Section 3.1, Proposition 2, the the clock rate dilation (i.e., the time-related component of the GRT curvature) is a consequence of the length dilations. This means that the information which specifies the length dilations implies the time-related component of the GRT curvature.

\section{Space(-time) dynamics}

The dynamics of spacetime is triggered by the minimal sources, called "quantum objects". With each update cycle of the system state a new space change action starts at each quantum object. The space changes propagate from the quantum objects through the whole space in steps according to the update cycles of the physics engine. In support of a local causal model, with each update cycle, the space changes propagate only to (part of) the neighboring space points. The propagating space changes always have definite directions at each space point, from the "in-connections" to the "out-connections" of the space point. The out-connections of space point sp, at a given update cycle $i$, are in-connections of some neighbor space points of sp with the subsequent update cycle $i+1$.

The directions of space changes, i.e., the identification of in/out-connections, are determined by the $\Delta$ curvature attribute of the space point connections. For a given space point, only part of the connections can be in-connections, which means connection. $\Delta$ curvature $>0$. The remaining connections of the space point are out-connections.

The overall process of space change propagation is specified as

Specification 1. spaceprogression ()$:=\{$

FOR ( all space points $\left.s p_{i}\right)\{$

IF ( inconnections $\left(s p_{i}\right)\{$

propagateOUT $\left(s p_{i}\right)$; 


\subsection{The emergence of space from a single source}

The space that emerges from a single source represents a Schwarzschild metric. In the causal model, the large-scale space object emerges by the successive addition of surface layers to the initial space object.

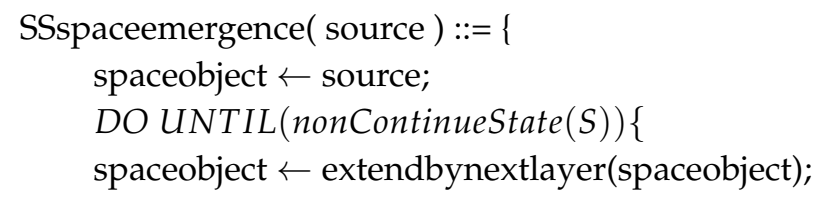

For the refinement of the above space emergence process, answers to the following questions have to be provided:

1. What are the elementary units of space?

2. How does the initial space object look like?

3. What is the detailed algorithm for extendbynextlayer(spaceobject)?

\subsubsection{The elementary units of space}

The elementary structure of space, including the elementary units of space, have already been described in Section 3.2. In the proposed model, the elementary units of space are the space points together with their connections to neighbor space points (see Definition 1). The number of connections (and thus the number of neighbor space points) of a given space point must be large enough to span the complete three-dimensional space. It should be small enough to enable a moderate growth of the number of space points with the chosen algorithm of the space emergence process. In the model, a typical space point has 14 connections (see Fig. 2):

- source connection: one connection towards the source of the emerging space,

- target connection: one connection in the primary emerging direction,

- surface connections: four connections in the plane that is perpendicular to the source connection (S1, S2, S3, S4 in Fig.2),

- four connections in between the source connection and the surface connections (A1, A2, A3, A4 in Fig.2),

- four connections in between the target connection and the surface connections (B1, B2, B3, B4 in Fig.2).

\subsubsection{The initial space object}

There are several alternatives for the initial space object from where the emergence of space and the propagation of gravitational space dynamics may start. Fig. 3 shows a number of alternatives investigated by the author. The simplest solution would be to have the space emergence process, starting from a single tetrahedron (case (a) in Fig. 3) or a double-tetrahedron (case (b) in Fig. 3) . However, more symmetrical initial space objects, such as case (c) or case (d) enable the early emergence of a symmetrical larger space object through simple space extension algorithms. For the present model of spacetime dynamics the initial space object is a single space point surrounded by 14 neighbor space points and the respective connections. The 14 neighbor space points, together with the interconnections among them represent a spherical surface - the initial surface from where the space emergence starts (case (d) in Fig. 3).

\subsubsection{The space expansion algorithm- extendbynextlayer (spaceobject)}

As described above, space emergence from a single source is a continuous process where each system state update cycle of the causal model adds another layer of space to the existing space object. 


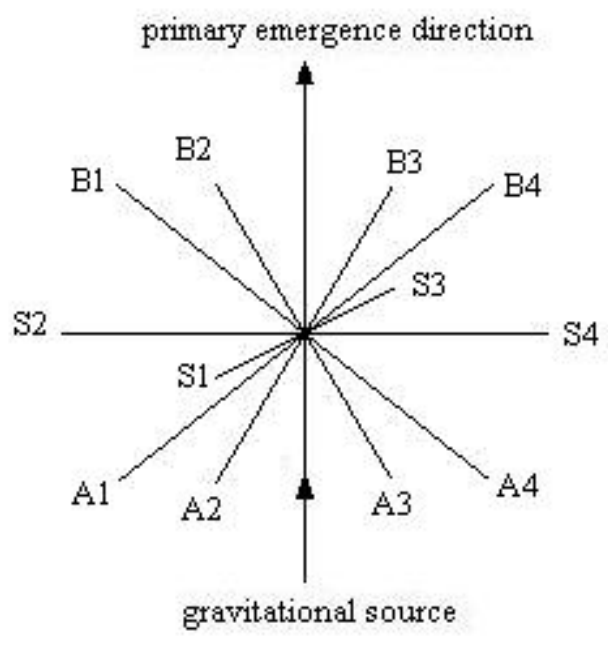

Figure 2. The 14 standard connections of a space point.

Table 1. Layers of space expansion, constant surface $\Delta r=1.0$

\begin{tabular}{|c|c|c|c|c|c|}
\hline $\begin{array}{c}\text { Layer } \\
\text { number }\end{array}$ & $\begin{array}{c}\text { surface } \\
\text { triangles, kt }\end{array}$ & $\begin{array}{c}\text { surface } \\
\text { points, kp . }\end{array}$ & $\begin{array}{c}\text { total } \\
\text { points, kpt }\end{array}$ & $\begin{array}{c}\text { radius, } \\
r_{i}\end{array}$ & $\begin{array}{c}\text { av. edge } \\
\text { length, L }\end{array}$ \\
\hline \hline 0 & 12 & 8 & 8 & 1.00 & 1.63 \\
\hline 1 & 36 & 20 & 72 & 2.00 & 1.72 \\
\hline 2 & 108 & 56 & 228 & 3.00 & 1.55 \\
\hline 3 & 324 & 164 & 660 & 4.00 & 1.22 \\
\hline 4 & 972 & 488 & 1956 & 5.00 & .88 \\
\hline$\ldots$ & $\ldots$ & $\ldots$ & $\ldots$ & $\ldots$. & $\ldots$ \\
\hline 12 & 6377292 & 3188648 & 12754596 & 13.00 & $\ldots$ \\
\hline 13 & 19131876 & 9565940 & 38263764 & 14.00 & $\ldots$ \\
\hline 14 & 57395628 & 28697816 & 114791268 & 15.00 & $\ldots$ \\
\hline$\ldots$ & $\ldots$ & $\ldots$ & $\ldots$ & $\ldots$ & $\ldots$ \\
\hline $\mathrm{i}$ & $3 \cdot k t_{i-1}$ & $k p_{i-1}+k t_{i-1}$ & $k s_{i}+3 k p_{i-1}$ & $(i+1) 100$ & \\
\hline
\end{tabular}

This means, with each expansion step $s t_{i}$ a number $k p_{i}$ of new space points is generated. The new space points are interconnected with their respective neighbor space point, forming $k t_{i}$ surface triangles. Various kinds of space expansion algorithms are possible. The key differentiating parameters for the alternative space expansion algorithms are the growth factor gp of the number of surface space points (i.e., $k p_{i}=g p \cdot k p_{i-1}$ ) and the related growth factor gt of the number of surface triangles (i.e., $\left.k t_{i}=g t \cdot k t_{i-1}\right)$. Table 1 shows the major parameters for an example space emergence algorithm that starts with an initial space object with 12 surface triangles (case (c) in Fig. 3). The surface growth factor $g t=3$, i.e., $k t_{i}=3 \cdot k t_{i-1}$. The number of surface space points increases by the number of surface triangles, $k p_{i}=k p_{i-1}+k t_{i-1}$.

Further parameters shown in Table 1 are the total number of space points, the radius $r_{i}$ of the surface and the average edge length, $\mathrm{L}$ of the surface triangles. The average edge length, $\mathrm{L}$ is the length measured by the author's computer simulations and these computer simulations and the length measurements assume Euclidean space. However, the space emergence process of the model of spacetime dynamics has to generate curved space that adheres to Schwarzschild metric, with length dilations in accordance with the Propositions 1, 2 and 3. Especially, Proposition 3 says that $L_{\text {connection }}$ is constant. With the example shown in Table $1, L_{\text {connection }}=\Delta r=1.0$. This means that the circumference of a surface, if curved space and $L_{\text {connection }}=1.0$ is assumed, depends solely on the number of surface space points, $k p_{i}$. The number of surface space points, $k p_{i}$ for a surface $S_{i}$ is determined by the space expansion algorithm. For the proposed model of spacetime dynamics, a curved space with length 


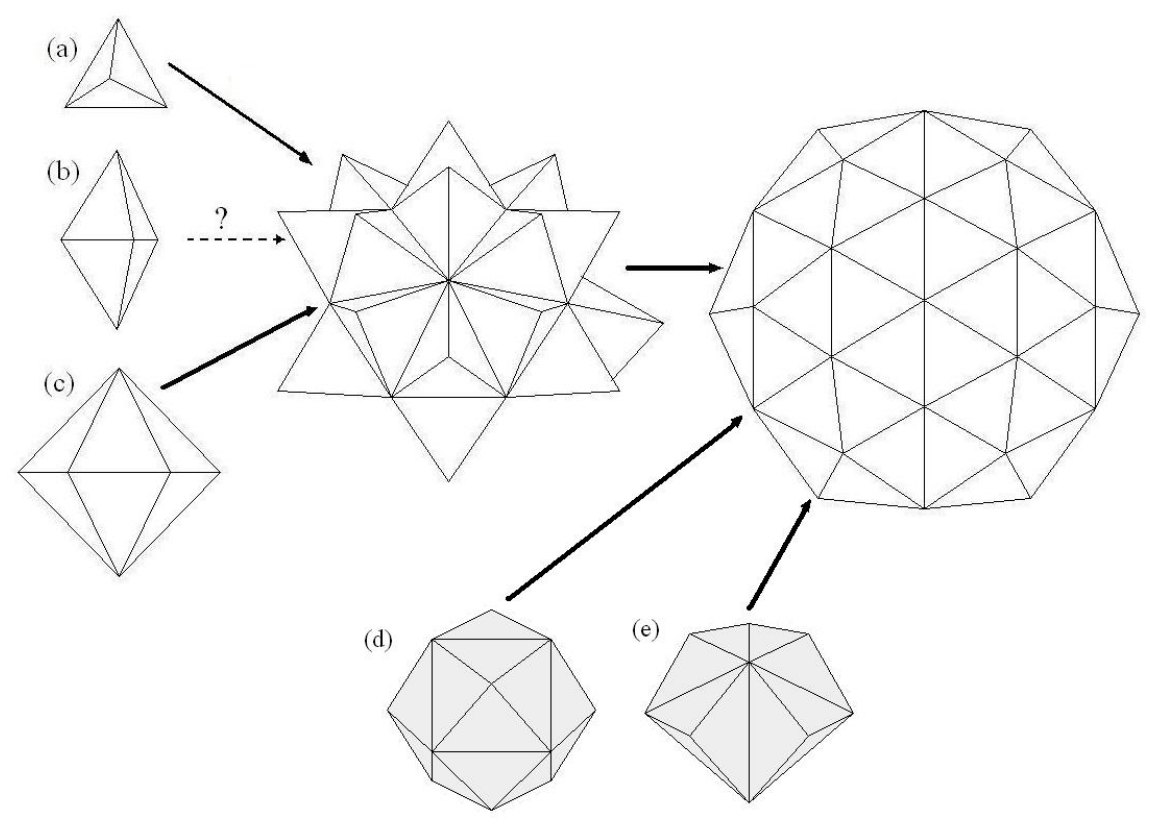

Figure 3. Alternative initial space elements.

dilations according to $F_{1}$ at the surfaces (see Eq. 2) has to emerge. This can only be achieved with a decreasing growth factor gp. The space expansion algorithms that have been investigated by the author showed that with the proposed model, GRT compatible space expansion algorithms are feasible. However, unless the algorithm gets unnaturally complex, occasional inhomogeneities seem to be unavoidable. In particular at the very small scale, i.e., near the minimal gravitational sources, it appears to be difficult or impossible to preserve the GRT compatible behaviour. ${ }^{10}$

\subsection{The propagation of space changes caused by multiple sources}

The assumption that space changes start at the minimal sources implies that the aggregation of space changes from many sources is the normal case. The model of the propagation of space changes that are caused by multiple sources is based on the single-source propagation (Section 4.1). The aggregation of the single-source propagations has to be accomplished by a local causal process, i.e., by a series of aggregations of neighboring space changes. Only long range, this dynamical process, can achieve overall gravitational space changes (i.e., curvature changes) that are compatible with the predictions of GRT and Newtonian dynamics.

To simplify the description, in this article, "multiple sources" is initially equated to "two sources". In simple cases, the treatment of many sources can be performed by a series of two source propagation processes.

For the overall two-source propagation process, three phases can be distinguished:

- Phase-1, the phase where the changes from the two sources propagate independently.

- Phase-2, the phase where the changes start to overlap and therefore have to be aggregated.

- Phase-3, the phase where the aggregated changes propagate like single source changes.

10 The surrender of perfect GRT compatibility at the very small scale may ease the provision of a causal model of the dynamics of QT/QFT (see Section 5) and avoids singularities that occur with the differential equations of GRT. 


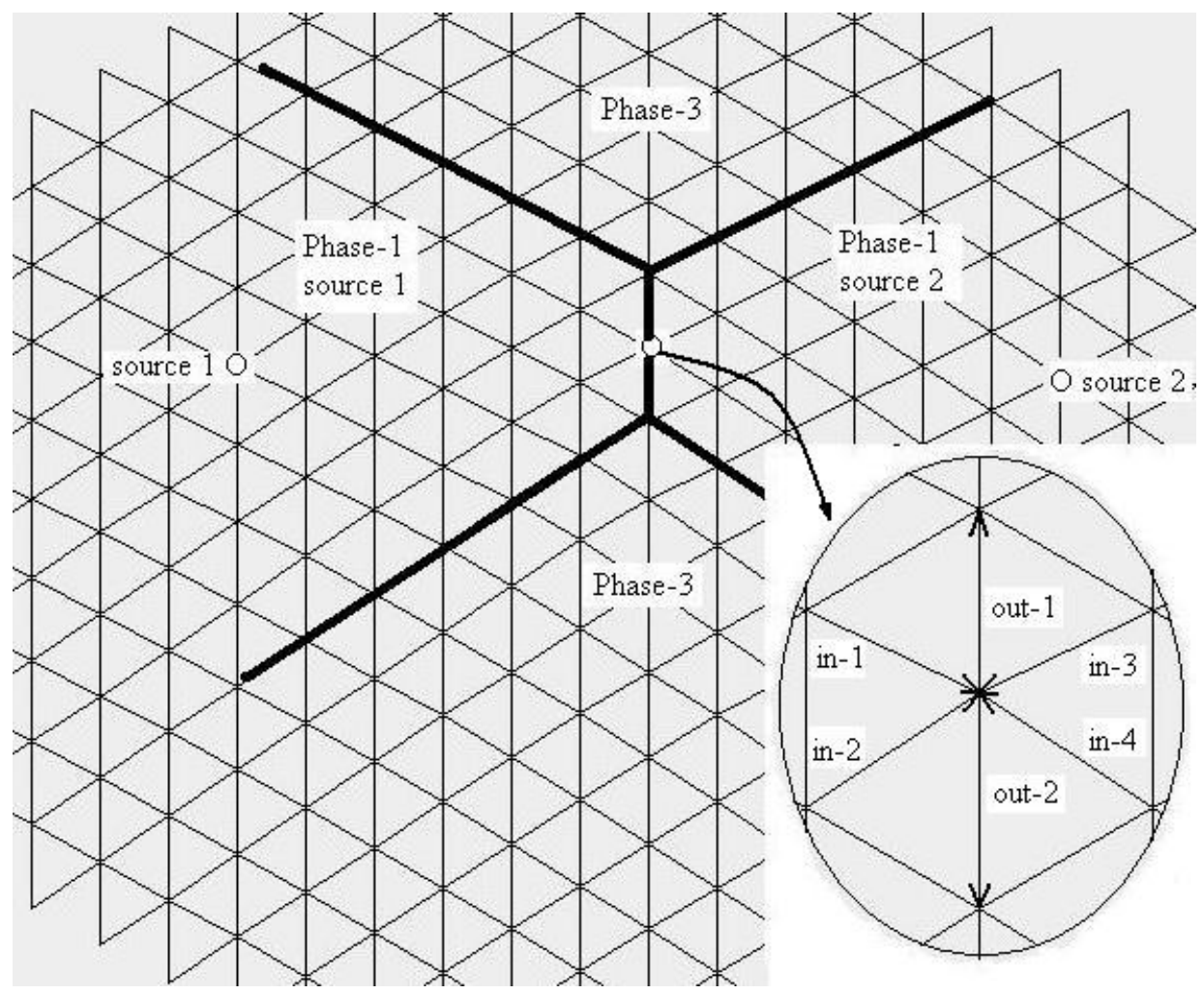

Figure 4. Propagation of space changes caused by 2 sources.

11 Notice that the 2-dimensional representation in Fig. 4 is a simplification which is misleading with certain more detailed considerations.

Fig. 4 shows an example snapshot in two dimensions, with the areas that are covered by phase- 1 and phase-3 roughly indicated. ${ }^{11}$

A major assumption of the proposed model is that the propagation that occurs at a space point sp has a definite (consolidated) in-direction and the same (overall) out-direction. The consolidated in-direction is the vector sum of the multiple in-connections. The overall out-direction is distributed over the multiple out-connections.

\subsubsection{Phase-1:}

The propagation of space changes prior to the points where the changes meet is exactly the single source propagation described in Section 4.1.

\subsubsection{Phase-2:}

When the space changes originating from (two) different sources meet at space point $\mathrm{sp}$, the changes that arrive from $n$ space point connections $(n \geq 2)$ are summarized into a single out-vector. The out-vector is then distributed to the out-connections (see Fig. 4, the magnifying glass area). If there are no out-connections left - i.e., if all connections of sp are in-connections - the weakest in-connection(s) are taken as out-connection(s).

\subsubsection{Phase-3:}

After the changes from the multiple sources are summed up, the further common propagation of the space changes continues like the single-source propagation (Section 4.1). As a special case, 
the phase-3 propagation may collide with phase- 1 propagation from one of the two sources. With the proposed model of spacetime dynamics, the collision of space changes is handled like a phase- 2 propagation, described above.

Compatibility with classical, i.e., Newtonian dynamics evolves during phase-3. The compatibility with classical dynamics is reflected in mainly the following items:

1. It is valid to assume an aggregated mass $M_{a g g r}$ that represents the aggregation of the masses of the sources of the space changes.

2. It is valid and possible to identify a position in space where $M_{a g g r}$ is assumed to be located. The position is usually called the "center of mass".

3. The (single) aggregated mass $M_{a g g r}$ is the sum of the masses of the sources of the space changes. $M_{\text {aggr }}=M_{1}+\ldots+M_{n}$.

Only when the propagation of space changes reaches a certain distance $r$ from the center of mass that the aggregated mass $M_{a g g r}(r)$ can be equated to the sum of the masses of the sources.

\subsubsection{Aggregation of space dynamics from $n \gg 2$ sources}

The above-described model of the space dynamics aggregation from two sources, with the three aggregation phases shows that compatibility with classical dynamics will only evolve at the end of phase-3. Prior to that stage, inhomogeneities, i.e., areas where only a subset of the gravitational source participates in the aggregation, will occur (and will not disappear during the continued propagation of space changes). If the aggregation of space dynamics applies to $n \gg 2$ sources, further inhomogeneities may exist, depending on the distribution of the sources within the space. If the distribution of the sources establishes gravitational sub-clusters such as solid bodies, planets or stars, where it is possible to assign an aggregated mass $M_{a g g r}$ and a center of mass, the sub-clusters may represent a gravitational source at the next higher level.

\section{The dynamics of quantum fields}

The model that is roughly described in the following is based on three types of work:

1. The causal model of spacetime dynamics described in Sections 3 and 4 constitutes the base with respect to the underlying spacetime structure and dynamics.

2. Further works that influenced the causal model described in the following is known under the names spin networks (see [17]), spin foam (see [18]) and causal fermion systems (see [19]). The coupling of the dynamics of space (e.g., the propagation of space changes) with the dynamics of quantum fields and particles is an idea that has already been pursued with causal fermion systems.

3. In [1] and [3] a causal model of QT/QFT is proposed where the physics of QT/QFT is confined in "quantum objects". For the refinement and an improved foundation of the model described in [1] and [3], a causal model of spacetime dynamics was felt to be required. The causal model of spacetime dynamics described in Sections 3 and 4 has been developed with the goal to provide this.

\subsection{Mapping of the dynamics of quantum fields to the dynamics of spacetime}

The refined causal model of QT/QFT can be summarized as follows:

A quantum object is a composite object consisting of 1 to $n$ particles. From the external point of view, i.e., for other quantum objects that may interact with it, the quantum object appears as a single object because for a certain time span it has associated well-defined, though possibly varying non-deterministically quantum-object-global attributes.

Definition 2. quantumobject $:=\{$ globalquantumobjectattributes; 


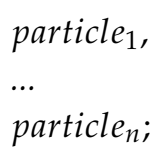

The lifetime of a quantum object, i.e., the time span for which the quantum object may be viewed as an entity with its specific attributes, depends on (1) the internal processes within the quantum object and on (2) possible interactions with other quantum objects (e.g., measurements or scatterings). A (semi-) stable quantum object, i.e., an object with a longer than a minimal lifetime, it can only occur if the internal process that involves the quantum objects particles is a (semi-) stable process, which means a process with a repetitive system state. A (semi-stable) process with a repetitive system state can only be achieved, if the spatial relationships among the components of the quantum object do not vary too much.

This leads back to the underlying model of spacetime dynamics. With the model of spacetime dynamics described in Sections 3 and 4, spacetime dynamics starts at the quantum object. This means that the spacetime curvature is maximal near the quantum object and within the quantum object. With the following proposition, a repetitive system state, and thus a semi-stable internal process, and thus a semi-stable quantum object is achievable.

Proposition 4. The dynamics of the quantum fields, including the external and internal dynamics of quantum objects, is an attachment to the spacetime dynamics described in Sections 3 and 4.

The internal dynamics of quantum objects and the dynamics of interactions between quantum objects are described by paths in space. The paths are comparable to the paths known from QFT, i.e., the paths of (virtual) particles in position space. Proposition 4 means that the paths of (virtual) particles follow the connections between space points. At each space point reached by the propagating space changes, the paths from the in-connections are joined and subsequently split and distributed to the out-connections. In QFT, the join/split operations are expressed in terms of creation and annihilation operators. There is, however, a significant difference between the creation/annihilation operators of QFT and the join/split operation performed at the space points of the proposed causal model. The operator combination of QFT (normally) applies to three operations (two creates and one annihilate or one create and two annihilate). The join/split operations of the causal model of the dynamics of quantum fields apply to the totality of the $\mathrm{n}$ space point connections (e.g., $n=14$ ). The in-connections are all joined together and the split affects all out-connections. To maintain compatibility with the Feynman rules of QFT, the following rule is established for the causal model of spacetime dynamics and QT/QFT:

Proposition 5. At most two in-connections and two out-connections may be assigned to (virtual) fermions; the remaining connections are assigned to (virtual) bosons.

The utilization of the complete set of in/out connections for the join/split operation on (virtual) particle paths delivers the equivalent to the superposition of paths which in QFT is expressed by the path integral. In standard QFT (see [16]), the path integral is written as

$$
K(b, a)=\int_{a}^{b} e^{(i / \hbar) S[b, a]} D x(t) .
$$

The discreteness of the model parameters (space, time and paths) may results in slight incompatibilities to standard QFT at large scale. It results in significant incompatibilities at very small scale. The discreteness of the model parameters in conjunction with the local causal model eliminates the need for renormalization (if a suitable algorithm for the assignment of in/out connections is applied). 


\subsection{Generalized spin networks}

With spin networks (see [17]) the connections (i.e., line segments) within the network are attributed by "spin numbers". Special rules define the computation of the spin numbers of out connections as a function of the spin numbers of the in connections. A given spin network thus defines possible paths of state transitions including possible final result states. A spin network is called closed, if line segments are all joined at vertices. A line segment (i.e., connection) of the spin network represents the spin of an elementary particle or of a compound system of particles, i.e., of a quantum object.

In Section 4, the propagation of spacetime changes is also described in terms of in/out connections of the space points. Also, in the causal model of QT/QFT described in [3], Feynman diagrams are mapped to the in/out connections and to split/join operators as with spin networks. The following generalization of the spin networks is therefore obvious.

The "generalized spin network" (GSN), is a network where the connections represent (virtual) particle types. As with the spin networks, the intersections of the line segments (i.e., the vertexes) represent split/join operators and the GSN is called a closed GSN, if line segments are all joined at vertexes. A specific GSN corresponds to a set of 1 to $n$ start particles of specific particle types. For example, a GSN containing two start particles can be associated with QFT scatterings and the pertinent Feynman diagrams. Generally, a GSN can be viewed as the network of Feynman diagrams that are applicable to a specific set of elementary particles. This means, the GSN, like a Feynman diagram, represents possible paths of state transitions. The join/split operators of a GSN specify for a given set of in connections the possible out connections together with the QFT rules for the determination of probability amplitudes.

While the proper GSN may be viewed as a tool for determination of the possible alternative state transition paths and the alternative outcomes of a set of particles, it may also be utilized for the determination of the multiple actual paths taken in a causal model of QT/QFT. For this purpose, actual paths with a definite (virtual) particle type and related attributes (e.g., spin) and probability amplitudes are assigned to the available connections of the space points reached during the propagation of spacetime changes. If the GSN is a closed GSN and in addition further physical conditions are satisfied, the process of state transitions may result in a repetitive loop until external influences disturb the process. The GSN together with the specific parameter settings then represents a stable composite quantum object.

The GSN has much similarity with the spin foam (see [18]).

\subsection{Collective behaviour}

One of the objectives of the causal model presented in this article is that the model should be a local causal model. The target space-point-locality is damaged by the inclusion of composite quantum objects with object-global attributes (e.g. mass and spin) and instantaneous processes (e.g., collapse of the wave function and entanglement), if it is not possible to break down the formation of the composite objects and the related non-local effects to space-point-local state transitions. In the causal model of QT/QFT described in [2], the non-local effects are explained by the collective behaviour of spacetime elements. Based on the causal model of spacetime dynamics described in Sections 3 and 4 and the concepts of the GSN, the model described in [2] can now be refined as follows.

The formation of (semi-) stable quantum objects (elementary as well as composite quantum objects) is a collective behaviour process that is

1. guided by the applicable closed GSN,

2. executing in a small area of curved space that represents the kernel of a gravitational source.

Guidance by the applicable GSN is generally assumed with QFT processes. In order to create a (semi-) stable quantum object, however, the GSN must be a closed GSN and the system must reach a "repetitive 
state". A repetitive state is a system state which, for a given GSN, has a high probability to recur. ${ }^{12}$ In general, the probability of the repetitive state can only be high if the spatial relationships, such as the distances between the involved particles, do not change too much, i.e., if the involved particles are confined in a small area of space. In the model of spacetime dynamics, where it is assumed that the changes of space curvature start already at the minimal sources (i.e., at the particles), space curvature around a set of particles is extremely high, forming a kind of lacuna.

As the described collective behaviour process represents a model for the emergence of quantum objects and the related quantum-object-global attributes, the disturbance of this collective behaviour process provides a possible model for the instantaneous non-local QT/QFT processes such as particle decay, the collapse of the wave function, and decoherence. The model which describes the emergence of a quantum object as a collective behaviour process has much similarity with G. Groessing's proposal to explain the emergence of a quantum system as a self-organization process (see [20]).

\subsection{Example: Scattering in quantum electrodynamics}

In quantum electrodynamics (QED), the operator equation for the creation and annihilation of the field has the form (see [21]):

$$
H_{W}(x)=-e N\left\{\left(\bar{\psi}^{-}+\bar{\psi}^{-}\right)\left(/ A^{+}+/ A^{-}\right)\left(\psi^{+}-\psi^{-}\right)\right\}_{x}
$$

where $\psi^{+}, \psi^{-}, \bar{\psi}^{-}, \bar{\psi}^{-}, / A^{+}, / A^{-}$are the creation and annihilation operators for electron, positron and photon. This leads to the eight possible first order Feynman diagrams shown in Fig. 5. For a real
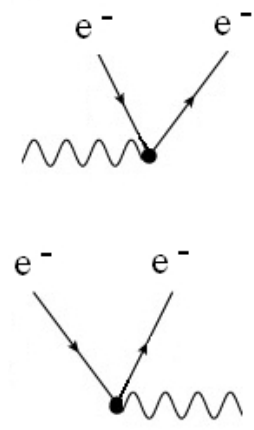
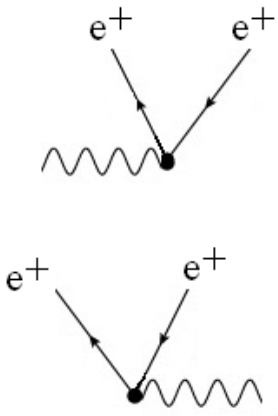
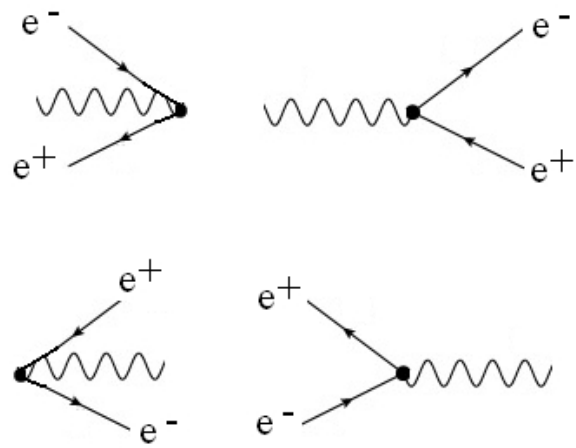

Figure 5. QED first order diagrams.

specific QFT scattering process, such as Bhabha scattering $\left(e^{+}, e^{-} \rightarrow e^{+}, e^{-}\right)$, the Feynman diagrams are combinations of the appropriate first order diagrams. For Bhabha scattering this leads to the two Feynman diagrams shown in Fig. 6.

With QFT in position space, the vertexes of the diagrams shown in Fig. 5 and 6 can be associated with space points. In the proposed model of spacetime, space points have associated connections to their immediate neighbor space points. As described in Section 5.1, the propagation paths of QFT (i.e., the lines of the Feynman diagrams) are mapped to the space point connections. As described in Section 4.1, a typical space point has 14 connections. This enables different strategies for the mapping of the three lines of the QED Feynman diagrams to the 14 space point connections. In Section 5.1 the overall strategy is described as the preservation of the number of fermion in-connections and fermion-out connections and the allowance of additional boson connections. This enables the types of QED space point connections shown in Fig. 7. ${ }^{13}$. The cases that correspond to the QED first order diagrams shown in Fig. 5 are the cases (1) to (3). Case (4) and case (5) increase the diversity

12 For a given GSN, there may exist multiple repetitive states and a repetitive state may allow a range of values for specific state components.

13 For practical purposes only part of the boson connections are shown in Fig. 7 


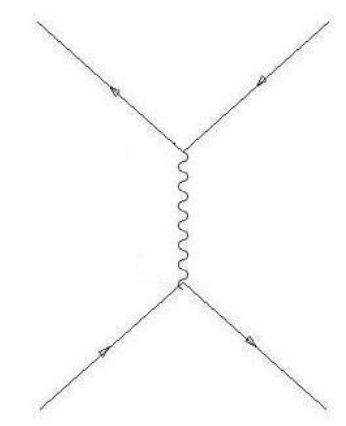

Bhabha Scattering Diagram A

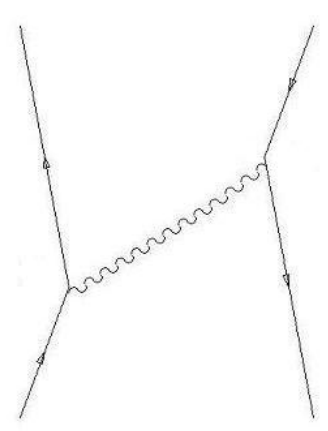

Diagram B

Figure 6. Feynman Diagrams for Bhabha Scattering.

of the possible fermion and boson paths. The more detailed strategy (i.e., algorithm) of the model
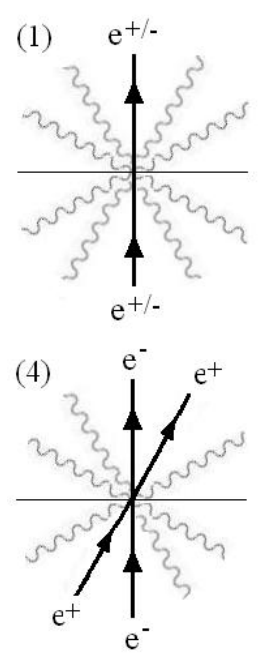

(2)
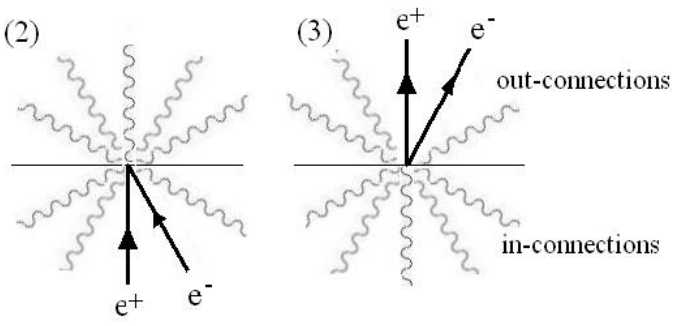

(5)

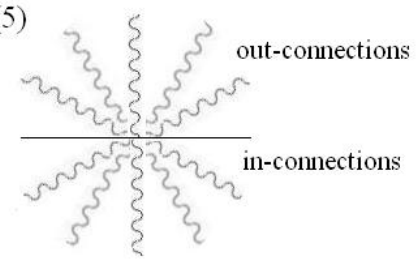

Figure 7. Possible QED connections of a space point.

determines (1) which of the connections of the space point are used as out connections, (2) which of the out connections are fermion connections and (3) the distribution of attributes such as momentum among the out connections. The respective algorithm contained in the present model is considered not to be the final algorithm. Further experimentation by use of computer simulations is in progress.

\section{Discussion}

\subsection{Local causal models, John Bell and David Bohm}

The work described in this article is presented in the form of a causal model. The availability of (or at least the feasibility of constructing) a causal model of an area of physics has been requested by the author in many articles (see, for example, [3]). The introduction of the term "local causal model" probably goes back to J. Bell when he formulated his famous Bell inequality and concluded that the refutation of the inequality in experiments prohibits the creation of a local causal model of QT (see [22]). It is probably not wrong to assume that J. Bell was convinced of the necessity of a (local) causal model of QT. This would also explain his admiration of David Bohm (see [23]) whose search for a deterministic model of QT may also be interpreted as the search for a causal model of QT (although a causal model does not necessarily has to be a deterministic model). In [23]), J. Bell wrote "I think 
that conventional formulations of quantum theory, and of quantum field theory in particular, are unprofessionally vague and ambiguous. Professional theoretical physicists ought to be able to do better. Bohm has shown us a way." Although Bells inequality has been refuted in Aspects experiment (see [24]) and Bohm's interpretation of QT did not get many supporters among QT physicists, their work and thinking influences QT physicists still until today with the attempts to overcome the many ambiguities, vaguenesses, and non-localities still contained in QT.

The authors attempt to construct a local causal model of QT/QFT (and later of the dynamics of spacetime) resulted in the experience that already, the attempt to construct a local causal model of an area of physics may uncover weaknesses and vaguenesses of a theory (see [9]). In addition, the goal of constructing a local causal model directs the selection of solutions towards specific solutions. For example, the space-time relationship described in Section 3.1. and the Propositions 1, 2 and 3 are all derived from the goal to construct a local causal model.

\subsection{The special role of time}

SRT and GRT have taught that space and time are integrated into spacetime. The major reason for taking this view is that in the laws and equations of SRT and GRT, time and space occur in combination, and the causal progression of the system state depends on the progression of the combination of both space and time. The causal model of spacetime dynamics presented in this article also implies a tight relationship of space and time, although with a different interpretation (see Section 3.1).

Nevertheless, there are also (good) reasons for not neglecting some fundamental differences between space and time. The major points where the concept of time assumed for the model described deviates from the time concept described (or implied) in some physics literature are:

\section{- Arrow of time}

The formal definition of a causal model (in general, not just for the model described in this article) assumes a constant direction in which time progresses, i.e., an arrow of time. Reverse progression of time or variable direction of time progression is just not supported by the model. The author believes that a causal model in general implies an arrow of time. In other words, a model that does not adhere to a unique constant direction of time would show more flexibility than nature shows in reality. The model would not be reality conformal.

- Time slices

With the goal of showing as much commonality as possible between space and time, some physics literature do not describe the extension of the time coordinate as differing from the extension of the space. In the formal definition of a causal model, the laws of physics that specify the state transitions can always access only the system state of the current point in time. It is not possible to access past or future time slices of system states. Models that would allow reference or even modifications of past or future system states are considered as (probably) not reality conformal and would be very complicated.

\subsection{Time dilation and/or length dilation?}

Both SRT and GRT predict, under specific circumstances, time dilation and/or length contraction. In textbooks covering SRT and GRT, it is not always clear whether (1) the two effects occur simultaneously, (2) the two effects are just two possible views from a non-local observer, or (3) there are cases where time dilation occurs (but no length contraction) and vice versa. For the proposed model of spacetime dynamics, length dilation is the primary effect. In the model, time dilation - more precisely, the clock rate dilation - is seen as a consequence of the length dilation. Length is a spatial attribute, while clock rate is a property of processes running in a causal subsystem. (In areas of space where there is no causal subsystem, there is no clock rate dilation, nor time dilation.) Despite the basic differences in the roles that time dilation and length dilation play (in the model), these functions are highly interrelated (see Section 3.1). 


\subsection{The general dependency of the clock rate on the length scaling}

The model that assumes that GRT/SRT-based length dilations generally imply, as a secondary effect, a proportional clock rate increase/decrease for the process that executes in the length-dilated area of space requires a further non-trivial assumption. The additional rule is Conjecture 3.1 in Section 3.1: "All physical processes can ultimately be broken down to length-related state changes, and changes in the length scaling, therefore, directly result in clock rate dilations of the affected process." If it were possible to identify a process that is not accompanied by some spatial state change ${ }^{14}$, and if it were possible to demonstrate that this process nevertheless adheres to GRT/SRT-predicted time dilation, this would prove that the model that assumes that time dilation is always a consequence of length dilation is wrong, or at least that it does not hold generally. The assumption that the rate of state change of a clock process and of arbitrary other processes that show a regular rate of state change depends in a predictable manner on the length scale of the space where the process executes is hard to believe. If the assumption could be confirmed, it would indicate another, even tighter relationship between time and space than is so far assumed with GRT.

\subsection{The role of computer simulations}

The development of the proposed causal model of spacetime and of QT/QFT has been accompanied by extensive computer simulations. Especially in areas where the causal model requires the determination of suitable algorithms computer simulations have been very useful. Mainly in the following areas the task of determining suitable algorithms has been supported by computer simulations:

1. The emergence of space from a single source with an increasing density of space points with increasing distance from the gravitational source such that maximum compatibility with GRT is provided (see Section 4.1).

2. The aggregation of space changes from multiple sources (see Section 4.2).

3. The assignment of in/out connections and of QFT particle types and particle attributes such that maximum compatibility with QFT is provided (see Section 5.4).

In all three areas, the simulations resulted in useful findings. ${ }^{15}$

\section{Conclusion}

The model of spacetime dynamics and of QT/QFT described in this article does not aim at providing another theory of the subjects. Rather, it has the goal of providing a special model, namely a causal model, of these subjects for which a generally agreed upon theories exist. However, it is not possible to derive a causal model of QT/QFT purely from existing QT/QFT. Nor is it possible to derive a causal model of spacetime dynamics purely from GRT. QT/QFT and GRT establish a powerful base for the development of the model, but supplementary statements and interpretations are required to construct a somewhat complete (local) causal model of these areas of physics and their combination. The described causal model is not claimed to be the only possible or valid model of the subjects. Alternative models, possibly focusing on specific aspects, are imaginable. With those features of the model that could not be directly derived from QT/QFT and/or GRT and where, therefore, new solutions had to be invented, it may turn out that the solutions of the present model have to be replaced by solutions that are in accordance with new experiments.

The two major items, where the proposed model deviates from the standard interpretations of GRT and QFT are:

14 An example could be the decay of particles.

15 The author does not yet consider the computer simulations as closed. Further computer simulations may result in further improvements. 
1. The assumption of the length dilation as the primary effect of space curvature that causes clock rate dilation as a secondary effect.

2. The assignment of additional bosonic create operators for the out-connections of space points (see Section 5).

Disregarding the uncertainties about the ultimate validity of certain details of the proposed model, there are nevertheless a number of findings that the author believes are worth noticing:

- For an area of physics, it is mandatory that the construction of models of the complete dynamics is feasible. The type of model that is best suited to describe the complete dynamics is the causal model. The lack of feasibility of constructing a causal model of a theory of physics may be considered as an indication of the incompleteness of the theory.

- As SRT and GRT show, space and time have to be viewed as integrated. The progression of time can be described only in connection with spatial state changes. The length scaling within space (including curvature) can only be described with reference to processes executing for a specific time interval. However, besides this fundamental tight relation between space and time, it is also necessary to point out the fundamental differences in the roles, structure, and properties of space and time.

Further work is required to refine the model and make the ideas more solid. Dealing with discrete space, time, and paths, refinements of the model may probably be achievable only with the help of computer simulations.

\section{Acknowledgments}

I am grateful to the Fetzer Franklin Fund who covers the article processing charge for this article.

\section{References}

1. Diel, H.H. A model of spacetime dynamics with embedded quantum objects. Rep. Adv. Phys. Sci. (2017) Vol. 1, No 3 1750010, https:/ / doi.org/10.1142/S2424942417500104

2. Diel, H.H. Collective Behavior in a Local Causal Model of Quantum Theory. Open Access Library Journal , (2017) 4: e3898. https://doi.org/10.4236/oalib.1103898

3. Diel, H. Quantum objects as elementary units of causality and locality (2016), http://arXiv:1609.04242v1

4. Loll, R.; Ambjorn J.; Jurkiewicz, J. The Universe from Scratch (2005), http://arXiv:hep-th/0509010

5. Loll, R.; Ambjorn J.; Jurkiewicz, J. Reconstructing the Universe (2005), http:/ /arXiv:hep-th/0505154

6. Ambjorn J.; Jurkiewicz, J.; Loll, R. Quantum Gravity, or The Art of Building Spacetime (2006), http://arXiv:hep-th/0604212

7. Thiemann, T. Loop Quantum Gravity: An Inside View. Approaches to Fundamental Physics. Lecture Notes in Physics. (2003) 721: 185-263.(2006) Bibcode:2007LNP...721..185T ISBN 978-3-540-71115-5. https://doi.org/10.1007/978-3-540-71117-9-10

8. Rovelli, C. Loop Quantum Gravity Living Reviews in Relativity. 1. Retrieved 2008-03-13. http://www.livingreviews.org/lrr-1998-1).

9. Diel, H. The completeness, computability, and extensibility of quantum theory, (2016) http://arXiv:1512.08720

10. 't Hooft, G. The Cellular Automaton Interpretation of Quantum Mechanics (2016) Springer, https://doi.org/10.1007/978-3-319-41285-6

11. Elze, H-T. Are nonlinear discrete cellular automata compatible with quantum mechanics?, J. Phys.: Conf. Ser. 631, 012069, (2015) http://arXiv:1505.03764

12. Fredkin, E. Digital mechanics: An informational process based on reversible universal cellular automata, Physica D 45, 254-270 (1990)

13. Diel, H. A Lagrangian-driven Cellular Automaton supporting Quantum Field Theory, (2015) http://arxiv.org/abs/1507.08277

14. Schutz, B.F. A First Course in General Relativity, (2009) Cambridge University Press, New York

15. Wikipedia on "Real-World Relativity: The GPS Navigation System" 
774 16. Feynman, R.P.; Hibbs A.R. Quantum Mechanics and Path Integrals, (2010) Dover Publication, New York

775 17. Penrose, R. The Road to Reality, (2004) Vintage Books, New York

776 18. Baez, J.C. Spin Foam Models, (1998) http://arXiv:gr-qc/9709052

777 19. Finster, F.; Kleiner, J. Causal Fermion Systems as a Candidate for a Unified Physical Theory". 778 Journal of Physics: Conference Series. 626 (2015): 012020. Bibcode:2015JPhCS.626a2020F

779 (http://adsabs.harvard.edu/abs/2015JPhCS.626a2020F), https://arxiv.org/abs/1502.03587

780 doi:10.1088/1742-6596/626/1/012020

781 20. Groessing, G. Emergence of Quantum Mechanics from a Sub-Quantum Statistical Mechanics, Int. J. Mod. 782 Phys. B, 28, 1450179 (2014), http:/ /arXiv:1304.3719 [quant-ph]

783 21. Mandl, F.; Shaw, G. Quantenfeldtheorie, (1993) AULA Verlag

784 22. Bell, J. S. On the Einstein-Podolsky-Rosen paradox. In Speakable and Unspeakable in Quantum Mechanics. (1987) 785 Cambridge UK: Cambridge University Press.

786 23. Bell, J. S. Beables for quantum field theory. In Speakable and Unspeakable in Quantum Mechanics. (1987) 787 Cambridge UK: Cambridge University Press.

788 24. Aspect, A.; Dalibard, J. and Roger, G. Phys. Rev. Letters (1982), 49, 1804-7 